Ciência e Natura, Santa Maria, v. 37 n. 4 set-dez. 2015, p.329-349

Revista do Centro de Ciências Naturais e Exatas - UFSM

ISSN impressa: 0100-8307 ISSN on-line: 2179-460X

\title{
ciênciaenatura
}

\section{PATIOS DE LA REGIÓN METROPOLITANA DE MARINGÁ (PARANÁ, BRASIL): APORTES A LA PLANIFICACIÓN Y A LA CONSERVACIÓN DE LA BIODIVERSIDAD}

\author{
BACKYARDS OF THE METROPOLITAN REGION OF MARINGÁ (PARANÁ, BRAZIL): CONTRIBUTIONS TO THE \\ PLANNING AND BIODIVERSITY CONSERVATION
}

Fabio Angeoletto, Camila Essy'1, Juan Pedro Ruiz Sanz'1, Ricardo Massulo Albertin², Frederico Fonseca da Silva ${ }^{3}$

\footnotetext{
${ }^{1}$ Grupo de Estudios en Ecología Humana, Urbana y del Paisaje de la Universidad Autónoma de Madrid.

2 Universidade Estadual de Maringá, PR, Brasil.

${ }^{3}$ Docente e investigador del Instituto Federal do Paraná, PR, Brasil.
}

\section{Resumen}

Patios urbanos tienen un potencial considerable para la conservación de la biodiversidad y la mejora de la seguridad alimentaria. Sin embargo, estos espacios no están bien planificados, y existe poca información sobre la diversidad de la flora presente en los patios de las diferentes clases sociales. Hemos cuantificado y comparado la diversidad vegetal de los patios de dos barrios de la zona metropolitana de Maringá (Paraná, Brasil), mediante el cálculo de índices de diversidad, correlaciones bivariadas y del potencial de plantíos, y trazamos directrices de planificación, con el objetivo de aumentar la presencia de la vegetación arbórea, fortalecer la seguridad alimentaria y contribuir a la conservación de la diversidad biológica, incluyéndose la conservación de las especies de plantas en peligro de extinción.

Palabras-clave: estudios interdisciplinares, ecología urbana, patios, biodiversidad, seguridad alimentaria, planeamiento urbanístico.

\footnotetext{
Abstract

Urban backyards have considerable potential for biodiversity conservation and the enhancement of food security. However, these spaces are poorly planned, there is little information on the diversity of flora present in the backyards of different social classes. We quantified and compared plant diversity of the backyards of two neighborhoods in the metropolitan area of Maringá (Paraná, Brazil), by calculating diversity indices, bivariate correlations and potential of plantations, and outline planning guidelines, with the goal of increasing the presence of woody vegetation strengthen food security and contribute to the conservation of biological diversity, including the preservation of endangered plant species.

Keywords: interdisciplinary studies, urban ecology, backyards, biodiversity, food security, urban planning.
} 
Hasta aquí he cantado el cultivo de los campos y el influjo de los astros; ahora, joh Baco!, te cantaré a ti, y contigo los silvestres arbolados y los tardíos renuevos del olivo. Asísteme, joh padre Leneo! Todo aquí está lleno de tus dones; por ti florece el campo cuajado de pámpanos otonales y la vendimia rebosa en las henchidas tinajas. Asísteme, joh padre Leneo!, y depuestos los coturnos, tiñe conmigo las desnudas piernas en el nuevo mosto.

Georgicon, liber II (Pvblivs Vergilivs Maro. Roma, 29 a.C.)

\section{INTRODUCCIÓN}

En 2007 la población urbana mundial superó la barrera del 50\%, alcanzando en América Latina y el Caribe el $79 \%$. El conjunto de impactos causados por la masiva urbanización mundial, en escala local, regional y global, es tajante (Pickett et al. 1997; Angeoletto, 2012). Según Vitousek (1994), la constante conversión de suelos en cultivos $\mathrm{y}$ ciudades es uno de los tres mayores impactos ambientales globales de origen humana, además de las crecientes concentraciones de $\mathrm{CO} 2$ en la atmósfera, y de otros cambios en los ciclos biogeoquímicos. En las próximas décadas, la urbanización será el impacto humano globalmente más significativo, principalmente en los trópicos, si profundos cambios en políticas y planificación de los usos de suelo no ocurrieren (Chapin III et al. 2000; Angeoletto, 2008). Efectivamente, la urbanización no planeada es una de las más significativas amenazas a la diversidad biológica (Schaedek et al. 2009).

En Brasil, el proceso de urbanización resultó en un desplazamiento de millones de personas desde las zonas rurales hacia las ciudades. En 1890 la población urbana de Brasil era cerca de 10\%. En pocas décadas, ese cuadro se invierte: hoy, 83\% de los brasileños viven en ciudades. En un primer ciclo, los emigrantes se han desplazado hacia las ciudades grandes. Desde mediados de los años 90, se inicia un según ciclo de urbanización en Brasil, con un aumento considerable del número de ciudades medias (con poblaciones entre 100.000 y 500.000 habitantes), de personas viviendo en ellas y del área ocupada por ellas. Se puede observar nítidamente una disminución del área ocupada por las metrópolis, mientras que el área ocupada por ciudades medias evoluciona, desde 1970 hasta 2000, de un 11,77\% para un 27,23\% del área total ocupada por las ciudades brasileñas. El número de ciudades medias también crece considerablemente en ese período, pasando de 40, en 1970, a 194, en 2000 (Carvalho, 2003). En 2010, el número de ciudades medias brasileñas llegó a 283, con aproximadamente el 25\% de la población de Brasil viviendo en ellas (Angeoletto, 2012).

Entre 2000 y 2010, esa expansión se mantuvo: mientras que ciudades pequeñas y grandes crecieron a tasas anuales semejantes $(1,06 \%$ y $1,07 \%$, respectivamente), las ciudades medias brasileñas crecieron $1,51 \%$ al año. Las tendencias actuales de la urbanización brasileña reflejan un fenómeno global. Contrariamente a la creencia general, el grueso del aumento de la población urbana mundial ocurre en ciudades medianas y pequeñas, cuyas capacidades de planificación y gestión generalmente son débiles (UNFPA, 2007).

En 2007, de los 3,3 mil millones de urbanitas, el 52\% vivían en ciudades con menos de 500 mil personas. Hasta 2025, esas ciudades van a absorber aproximadamente la mitad del crecimiento esperado de la población urbana global (United Nations, 2008). 
Además, los pobres constituirán una gran parte del futuro crecimiento poblacional urbano mundial (UNFPA, 2007). Pese a ello, la literatura sobre planificación urbana ha se concentrado en el estudio de grandes ciudades, y poca énfasis se ha dado a las ciudades pequeñas y medias. Con todo, solo un $12 \%$ de los ciudadanos viven en megaciudades [aquellas con más de 10 millones de vecinos (Montgomery, 2008)].

A su vez, por razones históricas, la biología de la conservación ha dividido el mundo en hábitats prístinos y degradados. No obstante, hace falta un cambio de mentalidades: la biología de la conservación debe volverse a los hábitats donde viven los seres humanos, y producir conocimiento sobre cómo dividir esos ambientes antropogénicos con especies silvestres (Rosenzweig, 2001). De hecho, aunque el porcentual de áreas protegidas esté aumentando mundialmente desde 1990, el número de especies amenazadas sigue creciendo (PNUMA, 2011), hechos que ponen de relieve la urgencia del desarrollo de mecanismos adicionales de conservación biológica.

\section{EL CARÁCTER INTERDISCIPLINARIO DE LA ECOLOGÍA URBANA Y SUS CONEXIONES CON LA PLANIFICACIÓN}

Según Grimm (et al. 2008), la ecología urbana es una ciencia que integra las teorías y metodologías de las ciencias naturales y sociales para investigar estándares y procesos de los sistemas ecológicos urbanos. Dow (2000) define la ecología urbana como un nuevo campo interdisciplinario en el cual la integración entre ecología y ciencias sociales resulta en una de las más activas intersecciones entre procesos biofísicos y sociales. El estudio de ecosistemas urbanos es un campo relativamente reciente de la ecología (Pickett y Grove, 2009).

Gracias a los avances tecnológicos, crecimiento poblacional y a una fuerte inclinación al consumo, nosotros somos hoy una fuerza ecológica global, capaz de afectar cada especie y ecosistema de la biosfera. Con todo, a pesar de esa contundente influencia, pocos son los estudios respecto a la ecología de los sistemas ecológicos urbanos (Botkin y Beveridge, 1997; Angeoletto, 2008; Grimm y Redman, 2004), y aún menos investigación sobre ecología de ecosistemas urbanos ha sido conducida en ciudades de países en desarrollo (Lubbe et al. 2010; Angeoletto, 2012).

A lo largo de casi todo el siglo XX, los ecólogos, en su mayoría, han evitado estudiar los ecosistemas urbanos. Consecuentemente, poco conocimiento ha sido producido con el objetivo de solucionar los problemas ambientales urbanos (Grimm et al. 2008). De hecho, la ecología se consolidó esencialmente a partir de estudios conducidos en ambientes prístinos. Sin embargo, el hueco de conocimientos sobre ecología urbana: 1) priva a la ecología básica de la comprensión de la más diseminada y extremada forma de intervención humana sobre la biosfera; 2) torna imposible a la ecología aplicada el acceso a opciones de gestión en los núcleos urbanos; 3) limita a la sociedad de proporcionar a los ciudadanos más calidad de vida, salud y bienestar (Pickett, 1997).

Desafortunadamente, ni la ecología urbana, ni la ecología en general, han sido aún plenamente incorporadas en los planteamientos habituales que presiden la planificación urbanística, territorial y económica (Terradas, 2001). No obstante, el design de ambientes urbanos ambientalmente más eficaces es la más importante demanda de planificadores y gestores, hecho que requiere un conocimiento más 
amplio respecto a los ecosistemas urbanos, y por extensión, el establecimiento de un foco interdisciplinario en la planificación (Haeuber y Ringold, 1998; Angeoletto, 2008; Angeoletto, 2012).

Poblaciones urbanas dependen de una vasta gama de servicios ecosistémicos, como la polinización, la regulación climática, y la absorción de carbono. Por otro lado, la manutención de esos servicios, o bien localmente, o bien regionalmente y globalmente, depende crecientemente de cómo se manifiestan los estándares de desarrollo de las ciudades. Usualmente, ciudades están ubicadas en ambientes-clave: próximas de ríos, estuarios, manglares y bosques. Por lo tanto, ciudades suelen desarrollarse en escenarios cruciales a la conservación biológica, hecho que pone de manifiesto la importancia de la planificación de urbes más amigables a la vida silvestre. La planificación adecuada de los sistemas ecológicos urbanos es tan importante a la conservación de la biodiversidad cuanto es el establecimiento de áreas naturales legalmente protegidas (Botkin y Beveridge, 1997; Angeoletto, 2012).

\section{QUEMANDO INCIENSO EN ALTARES CERCANOS: LOS QUINTAIS COMO ESPACIOS DE CONSERVACIÓN BIOLÓGICA}

Un adagio chino dice que es mejor ser bueno en tu propia casa que quemar incienso en un templo distante. Por la enorme influencia de las ciudades sobre la biosfera, la planificación de su crecimiento se ha convertido en uno de los más importantes desafíos del siglo XXI (Cohen, 2006), con reflejos obvios en la conservación de especies, ecosistemas y biomas, en ámbito global. En los patios urbanos, los objetivos de disminución de los impactos ambientales urbanos y de conservación de la diversidad biológica coinciden. La definición del término patios es variable en la literatura técnica. Gaston (et al. 2005) los definen como espacios privados adyacentes a las viviendas, y que pueden contener, en grados variados, céspedes, polígonos con vegetación ornamental y alimentaria, fuentes de agua, caminos, y a veces construcciones temporarias, como invernaderos. $\mathrm{O}$, sencillamente, se puede caracterizarlos como el área que ha quedado después de construida la vivienda, en un lote particular (Smith et al. 2006b), definición que hemos adoptado para nuestro estudio.

Aunque los patios sean aparentemente demasiado diminutos para que resulten biológicamente significativos, cuando sumados alcanzan un área de dimensión contundente (Gaston et al. 2005; Daniels y Kirkpatrick, 2006; Loram et al. 2007; Marco et al. 2008). De cierto, el área ocupada por los patios en los ecosistemas urbanos suele ser tajante. En la ciudad de Dayton, EEUU, el 19,5\% de su área está ocupada por patios (Daniels y Kirkpatrick, 2006). En Léon (Nicaragua) los patios constituyen el 86,2\% de la superficie de áreas verdes urbanas (González y Gómez-Sal, 2008). El área de céspedes en patios de viviendas en los EEUU está estimada en 16 millones de hectáreas, rebosando largamente cultivos agrícolas económicamente importantes como la cebada (5 millones de hectáreas), algodón (4,5 millones de hectáreas) y arroz (1,1 millón de hectáreas) [Robbins et al. 2001].

Hay un amplio reconocimiento de la importancia de los patios privados a la conservación de la biodiversidad (incluyéndose a preservación de especies amenazadas vegetales y animales), pero prácticamente no ha habido intentos de describir la composición y distribución de la diversidad biológica presente en los patios 
(Gaston et al. 2005; Thompson et al. 2005; Smith et al. 2006a; Loram et al. 2007; Angeoletto, 2008; Loram et al. 2008; Marco et al. 2008; Davies et al. 2009; Goddard et al. 2010; Siviero, et al. 2011). Los estudios de la flora urbana en general se concentran en fragmentos de bosques (Hope et al. 2003) aunque las especies cultivadas sean las dominantes en los ecosistemas urbanos, y de ellas poco se sepa (Marco et al. 2008).

\section{MATERIALES Y MÉTODOS}

ÁREA DEL ESTUDIO

La región metropolitana de Maringá (RMM) fue creada en 1998 por la Ley Estadual no 83/98, siendo compuesta por ocho municipios, en área de 2,2 mil $\mathrm{Km}^{2}$. Se encuentra localizada a $23^{\prime \prime} 25^{\prime} 38.29^{\prime \prime} S$ y $51^{\prime \prime} 56^{\prime} 06.32 \mathrm{O}$. Predominan en la región los latosuelos, principalmente el latosuelo púrpura distrófico. El clima es el subtropical húmedo mesotermico, con veranos calientes y lluvias concentradas en los meses de verano, pero sin una estación seca definida. La media de temperaturas de los meses más calientes es superior a los $22^{\circ} \mathrm{C}$, y la de los meses más fríos, inferior a $18{ }^{\circ} \mathrm{C}$ (Angeoletto, 2012).

Las ciudades brasileñas presentan una elevada heterogeneidad respecto a la clase social predominante en los diferentes barrios. Gradientes urbanos son esencialmente gradientes antropogénicos, producidos como una consecuencia del surgimiento de asentamientos humanos. Por su elevada complejidad, son gradientes indirectos, es decir, no es posible estudiarlos a través de transectos, como se hace en investigaciones de gradientes directos (McDonnel y Hahs, 2008; Lubbe et al. 2010).

Por ello, hemos optado por el uso de gradientes sociales, no lineales, abarcando un barrio de baja renta de la ciudad de Sarandi (el Jardim Universal), y uno de renta elevada en la ciudad de Maringá (el barrio Zona 02). Sarandi (población circa 83.000 habitantes) y Maringá (población circa 357.000 habitantes) son ciudades conurbadas con profundas diferencias de renta y escolaridad entre sus habitantes (Angeoletto, 2012). Este abordaje metodológico posibilita amalgamar datos sociológicos e ecológicos, permitiendo así el discernimiento de estándares de diversidad biológica entre distintas clases sociales, y la producción de informaciones con un gran potencial de aplicación en la gestión y planificación de sistemas ecológicos urbanos (Dow, 2000; Grimm et al. 2000; McDonnel y Hahs, 2008; Lubbe et al. 2010).

El barrio Jardim Universal está constituido por 571 viviendas (y por lo tanto, 571 patios), y está poblado por familias de baja renta. En el barrio Zona 02, son 895 viviendas ocupadas mayoritariamente por familias de clase media alta. Fueron sorteadas a través del software Statistica 7 un muestreo aleatorio de 230 viviendas en el Jardim Universal, y 261 viviendas en la Zona 02. El tamaño del muestreo seleccionado ha sido obtenido con un error de estimativa de un 5\% y confiabilidad de un $95 \%$. Para los barrios investigados, la tarea de sortear las viviendas fue precedida por una investigación, con mapas en escala 1:2000, para identificar los terrenos baldíos.

Identificadas las viviendas sorteadas en los mapas de los barrios, las familias eran visitadas, y utilizamos la técnica de la visita guiada (Florentino et al. 2007), donde un miembro de la familia era invitado a caminar por los patios, durante la entrevista, suministrando informaciones específicas sobre los usos de las plantas presentes. Las 
entrevistas se realizaron tras el entrevistado haber firmado una declaración de consentimiento, en la cual se consentía en participar del estudio y se autorizaba la divulgación de los resultados.

Los usos mencionados por el encuestado eran anotados. Las especies citadas fueron relacionadas en las siguientes categorías de uso: hortícolas, frutales, medicinales y ornamentales. En relación a su origen, las especies fueron clasificadas en exóticas o nativas. Terminada esa etapa, mensurábamos el área total de los patios, el área solado y el área no solado de los mismos. Todas las especies cultivadas directamente en el suelo eran cuantificadas en relación al número de individuos cultivados. No incluimos en la toma de datos individuos cultivados en macetas. La identificación del material botánico ha sido hecha en campo. Las especies cuya identificación no fue posible en campo fueran clasificadas en nivel de familia, género y especie en el Herbario de la Universidad Estadual de Maringá. El sistema taxonómico utilizado fue el APG III (The Angiosperm Phylogeny Group, 2009). La nomenclatura de los nombres científicos fue chequeada a través de bases de datos Plantminer (Siviero et al. 2011).

Todas las especies identificadas fueron chequeadas en la página web de la Red List de especies amenazadas de extinción de la Internacional Union for Conservation of Nature - IUCN (http://www.iucnredlist.org). Además de chequeadas en la Red List, las especies brasileñas también fueron verificadas en la lista de especies amenazadas producida y publicada en la página web del Ministerio del Medio Ambiente de Brasil (http://www.mma.gov.br/sitio), ambas accedidas en julio de 2014.

\section{MEDIDAS DE LA DIVERSIDAD BIOLÓGICA}

Para calcular y comparar la diversidad de especies vegetales entre los barrios investigados, cuantificamos la riqueza de especies de los barrios del estudio, destacando las familias con mayor número de especies. También hemos calculado el Índice de Valor de Preferencia, el Índice de Diversidad de Shannon-Wiener, el Índice de Dominancia de Simpson, y el Índice de Similitud de Morisita-Horn. Las fórmulas para los cálculos son las siguientes: índice de valor de preferencia: IVP\%=Abu-Rel\% + FreRel\%, donde $\mathrm{Abu}$-Rel\% es el número de individuos de una especie, dividido por el número total de individuos de todas las especies observadas en cada barrio, multiplicado por 100; y Fre-Rel\% es el número de viviendas en que una determinada especie ocurre, dividido por el número total de viviendas de la muestra, multiplicado por 100. El IVP apunta el porcentaje de patios donde ocurre una determinada especie vegetal, además valorando la frecuencia de la especie en los patios. Lo hemos calculado para los conjuntos de plantas hortícolas, frutales, medicinales y ornamentales.

Índice de Shannon-Wiener: $\left.H=-\sum(n i / N) \log n i / N\right)$ donde $n i=$ valor de importancia de cada especie (el número de individuos de cada especie) y $N=$ total de los valores de importancia. Índice de Dominancia de Simpson: $S=\sum n i[n i(n i-1) / N(N-1)]$, donde $n i=$ valor de importancia de cada especie; $N=$ total de los valores de importancia de las especies. A través de testes T de Student, hemos mensurado se las diferencias entre los 
valores de los Índices de Diversidad de Shannon (H) y Simpson (S) son significativas, para los barrios investigados. El Índice de Diversidad de Shannon-Wiener es usado para caracterizar a diversidad de especies en una muestra. El Índice de Dominancia de Simpson mide el grado en que la comunidad es dominada por una o pocas especies comunes.

El grado de similitud entre la flora de los patios de barrios fue calculada a través del Índice de Similitud de Morisita-Horn, de acuerdo con la fórmula Cmh $=2 \sum$ (ani . bni) / da + $d b)(a N) .(b N)$ donde $a N=$ número total de individuos del barrio $\mathrm{A} ; b N=\mathrm{n}^{\circ}$ total de individuos del bairro $\mathrm{B} ; a n i=\mathrm{n}^{\circ}$ de individuos de cada especie en el bairro $\mathrm{A} ; b n i=\mathrm{n}^{\circ}$ de individuos de cada especie em el bairro B; $d a=\sum a n i^{2} / a N^{2}$ y $d b=\sum b n i^{2} / b N^{2}$. Índices de Similitud de Morisita-Horn superiores a 0,75 apuntan una elevada similitud de especies entre dos áreas investigados, mientras que aquellos inferiores a 0,50 revelan una baja similitud de especies entre dos áreas. Por otro lado, cuanto menor la similitud entre dos muestreos, más elevada será la diversidad beta entre ellos.

Con el objetivo de verificar si el área libre (no solado) de los patios influye sobre el número de especies e individuos presentes, fueron calculadas correlaciones bivariadas entre el área libre de los patios y número de especies presentes; entre el área libre de los patios y el número total de individuos cultivados; y entre el área de los patios y el número de árboles cultivados. Con el objetivo de verificar si la edad de las viviendas (es decir, el número de años desde que la vivienda ha sido construida) influye sobre el número de especies e individuos presentes en los patios, fueron calculadas correlaciones bivariadas entre el área libre de los patios y número de especies; entre el área libre de los patios y entre el número total de individuos cultivados; el área de los patios y el número de árboles cultivados. Santos (2007) propone tres coeficientes de correlación, para distinguir las correlaciones bivariadas, cuando estas se verifican: fuerte positiva $(0,8 \leq \mathrm{r}<1)$; moderada positiva $(0,5 \leq \mathrm{r}<0,8)$; y flaca positiva $(0,1 \leq \mathrm{r}<0,5)$.

Finalmente, hemos cuantificado el número medio de árboles por patios de los barrios estudiados, y basados en ello, y en el suelo disponible para plantíos (área libre medio de los patios de cada barrio, multiplicado por el número de patios del barrio), hemos estimado el potencial de plantíos para los dos barrios del estudio. Para ese cálculo, hemos desarrollado la ecuación $P P=\left\{\left[s d\left(m^{2}\right) / 9 m^{2}\right]-n m a p\right\}$, donde: $P P=$ potencial de plantíos; $s d=$ suelo disponbible para plantíos; y nmap= número medio de árboles por patios, considerándose $9 \mathrm{~m}^{2}$ como el área adecuada al crecimiento de un plantón de árbol. No hemos considerado la vegetación herbácea y arbustiva en la cuantificación de los potenciales de plantíos, porque esos tipos botánicos no son excluyentes. Al contrario, patios con buena cobertura vegetal son aquellos que poseen un estrato herbáceo, seguido de un arbustivo y finalmente, de un arbóreo (Angeoletto, 2012).

\section{RESULTADOS}

Los patios de Jardim Universal presentan área libre medio de $108,3 \mathrm{~m}^{2}$, y de $164,4 \mathrm{~m}^{2}$ en el barrio Zona 02. El 9,1\% de los patios del barrio pobre de nuestro análisis están completamente encementados (y por lo tanto, sin vegetación) porcentual que baja a un solo 3,5\% en el barrio Zona 02. En ambos los barrios investigados, verificamos que las variables número de especies, número de individuos y número de árboles se correlacionan al 
área libre de los patios, y al tiempo de construcción (edad) de las viviendas, aunque flacamente (cuadro 1).

Cuadro 1. Correlaciones bivariadas

\begin{tabular}{|l|l|l|}
\hline Correlación bivariada & Jardim Universal & Zona $\mathbf{0 2}$ \\
\hline Área $x$ Número de especies & $0,4413, \mathrm{p}<0,0001$ & $0,4304, \mathrm{p}<0,0001$ \\
\hline Área $\times$ Número de individuos & $0,4789, \mathrm{p}<0,0001$ & $0,4229, \mathrm{p}<0,0001$ \\
\hline Área $\times$ Número de árboles & $0,4215, \mathrm{p}<0,0001$ & $0,4592, \mathrm{p}<0,0001$ \\
\hline Edad $\times$ Número de especies & $0,3281, \mathrm{p}<0,0001$ & $0,3863, \mathrm{p}<0,0001$ \\
\hline Edad $\times$ Número de individuos & $0,1544, \mathrm{p}<0,0001$ & $0,1913, \mathrm{p}<0,0001$ \\
\hline Edad $\times$ Número de arboles & $0,3716, \mathrm{p}<0,0001$ & $0,4009, \mathrm{p}<0,0001$ \\
\hline
\end{tabular}

Fuente: elaboración propia

Hemos cuantificado 151 especies cultivadas en el Jardim Universal, y 381 especies cultivadas en los patios del barrio Zona 02 (cuadro 2). En el Jardim Universal las familias más representativas en relación a la diversidad fueron: Asteraceae, 13 especies; Euphorbiaceae, 7 especies; Araceae, 7 especies, y Myrtaceae, 6 especies. En la Zona 02, las familias mas representativas en relación a la diversidad fueron: Araceae (17 especies); Asteraceae (17 especies); Arecaceae (14 especies) y Euphorbiaceae (13 especies). Especies exóticas son dominantes en la flora de ambos barrios. En cuanto a los usos, especies ornamentales predominan en los patios del barrio de clase media alta, mientras que especies utilitarias (es decir, medicinales, alimentarias y de producción de sombra) predominan en los patios del Jardim Universal (cuadro 3).

La diversidad de especies mensurada por el índice de Shannon-Wiener es más grande (cuadro 4), y significativamente diferente (cuadro 5) en los patios de los vecinos de clase media alta de la Zona 02, cuando comparada a la diversidad de flora encontrada en los patios de los vecinos pobres del Jardim Universal. El bajo índice de dominancia de Simpson (S) que hemos calculado, en ambos los barrios, indica una elevada riqueza específica. No hay una diferencia significativa entre los índices de dominancia de Simpson a lo largo del gradiente social investigado (cuadros 4 y 5 ).

Cuadro 2. Riqueza de familias, géneros y especies

\begin{tabular}{|l|l|l|l|}
\hline Barrio & Número de familias & Número de géneros & Número de especies \\
\hline Jardim Universal & 66 & 137 & 151 \\
\hline Zona 02 & 108 & 278 & 381 \\
\hline
\end{tabular}

Fuente: elaboración propia

Cuadro 3. Porcentaje de especies exóticas y ornamentales

\begin{tabular}{|l|l|l|l|l|}
\hline Barrio & $\begin{array}{l}\text { Número de } \\
\text { especies }\end{array}$ & $\begin{array}{l}\text { Porcentaje de especies } \\
\text { exóticas }\end{array}$ & $\begin{array}{l}\text { Porcentaje de especies } \\
\text { ornamentales }\end{array}$ & $70,1 \%$ \\
\hline Zona 02 & 381 & $77,7 \%$ & $19,2 \%$ & \\
\hline $\begin{array}{l}\text { Jardim } \\
\text { Universal }\end{array}$ & 151 & $82,8 \%$ & & \\
\hline
\end{tabular}

Fuente: elaboración propia 
Cuadro 4. Índices de Diversidade de Shannon y de Dominancia de Simpson

\begin{tabular}{|l|l|l|}
\hline Barrio & Índice de Shannon-Wiener (H) & Índice de Dominancia de Simpson (S) \\
\hline Zona 02 & 4,61 & 0,033 \\
\hline Jardim Universal & 3,79 & 0,048 \\
\hline
\end{tabular}

Fuente: elaboración propia

Cuadro 5. Significancia del test-t $(\mathrm{p}<0,05)$ para los Índices de Shannon y Simpson

\begin{tabular}{|c|c|c|}
\hline Barrios & (H) & (S) \\
\hline Zona 02 x Jardim Universal & SIGNIFICATIVO & NO SIGNIFICATIVO \\
\hline
\end{tabular}

Fuente: elaboración propia

Hemos encontrado en los patios investigados de los dos barrios, especies frutales que o bien no son comercializadas en la región metropolitana de Maringá (como por ejemplo Campomanesia xanthocarpa, Eugenia pyriformis, Psidium cattleianum, todas con bajos IVP's), o bien son muy escasamente comercializadas (Myrciaria cauliflora), constituyéndose de ese modo en fuentes importantes de germoplasma vegetal y de alimento. Algunas de esas especies poseen IVP's bastante expresivos, como Eugenia uniflora y Myrciaria cauliflora (en la Zona 02). Sin embargo, algunas especies, como las frutales Eugenia jambolana, Passiflora alata y Spondias purpurea ocurren apenas en el Jardim Universal (aunque con IVP's bajos), lo que demuestra que su contribución a la conservación de agrobiodiversidad no es despreciable. Las tres especies con mayores índices de valor de preferencia por usos, están relacionadas en el cuadro 6.

Cuadro 6. Índices de valor de preferencia por usos

\begin{tabular}{|c|c|c|c|}
\hline Uso y especie & $\begin{array}{c}\text { IVP\% } \\
\text { (Jardim Universal) } \\
\end{array}$ & $\begin{array}{c}\text { IVP\% } \\
\text { (Zona 02) }\end{array}$ & Uso y especie \\
\hline (Hort.) Allium fistulosum L. & 36,3 & 20 & Allium fistulosum L. (Hort.) \\
\hline (Hort.) Arachis hypogaea L. & 16,2 & 12,6 & Ocimum basilicum L. (Hort.) \\
\hline (Hort.) Manihot sculenta C. & 15,4 & 11,4 & Mentha piperita L. (Hort.) \\
\hline (Frutal) Citrus limon (L.) Burm. & 50,4 & 29 & $\begin{array}{r}\text { Myrciaria cauliflora (Mart.) O. } \\
\text { Berg } \\
\text { (Frutal) }\end{array}$ \\
\hline (Frutal) Mangifera indica L. & 36,2 & 27,5 & Citrus limon (L.) Burm. (Frutal) \\
\hline (Frutal) Citrus reticulata Blanco & 34,3 & 22,4 & Eugenia uniflora L. (Frutal) \\
\hline $\begin{array}{l}\text { (Med.) Coleus barbatus (Andrews) } \\
\text { Benth. }\end{array}$ & 15,4 & 16,1 & $\begin{array}{r}\text { Coleus barbatus (Andrews) } \\
\text { Benth. (Med.) }\end{array}$ \\
\hline (Med.) Aloe vera Mill. & 14,7 & 9,9 & $\begin{array}{r}\text { Cymbopogon citratus (DC.) Stapf } \\
\text { (Med.) }\end{array}$ \\
\hline (Med.) Costus spicatus (Jacq.) Sw. & 8,2 & 7,6 & $\begin{array}{r}\text { Costus spicatus (Jacq.) Sw. } \\
\text { (Med.) }\end{array}$ \\
\hline (Orn.) Impatiens walleriana Hook. f. & 23,2 & 48,8 & Duranta repens L. (Orn.) \\
\hline (Orn.) Rosa L. & 22,5 & 35,5 & Ixora coccinia L. (Orn.) \\
\hline (Orn.) Duranta repens L. & 22,4 & 29,5 & $\begin{array}{r}\text { Dypsis lutescens } \\
\text { (H. Wendl.) Beentje \& J. Dransf. } \\
\text { (Orn.) }\end{array}$ \\
\hline
\end{tabular}

Fuente: elaboración propia

Hemos cuantificado el índice de similitud de especies de Morisita-Horn en $\mathbf{0 , 4 8 1}$, lo que indica una gran diferencia en las floras cultivadas a lo largo del gradiente social 
estudiado, y, por lo tanto, una elevada diversidad beta, cuando comparadas las floras de las distintas clases sociales.

Cuantificamos el número medio de árboles en 4,4 árboles por patio en el Jardim Universal y en 5,2 árboles por patio en la Zona 02; y el número medio de individuos arbustivos en 6,7 y 31,5, para el Jardim Universal y Zona 02, respectivamente (cuadro 7). Conocido el potencial de plantíos medio de los patios (o sea, cuántos árboles más podrían ser introducidas por patio), hemos calculado cuantos árboles frutales podrían ser plantados, de acuerdo con el número de patios de los barrios (cuadro 8).

Cuadro 7. Número medio de árboles y arbusto por patio

\begin{tabular}{|l|l|l|}
\hline Barrio & Número medio de individuos arbóreos & Número medio de individuos arbustivos \\
\hline Jardim Universal & 4,4 & 6,7 \\
\hline Zona 02 & 5,2 & 31,5 \\
\hline
\end{tabular}

Fuente: elaboración propia

Cuadro 8. Potenciales de plantíos

\begin{tabular}{|l|c|c|c|c|c|}
\hline \multicolumn{1}{|c|}{ Barrio } & $\begin{array}{c}\text { Área } \\
\text { medio } \\
\mathbf{( m}^{\mathbf{2}}\end{array}$ & $\begin{array}{c}\text { Número de } \\
\text { patios }\end{array}$ & $\begin{array}{c}\text { Número medio } \\
\text { de árboles por } \\
\text { patio }\end{array}$ & $\begin{array}{c}\text { Potencial de } \\
\text { plantíos de árboles } \\
\text { por patio }\end{array}$ & $\begin{array}{c}\text { Potencial de } \\
\text { plantíos de árboles } \\
\text { - total }\end{array}$ \\
\hline $\begin{array}{l}\text { Jardim } \\
\text { Universal }\end{array}$ & 108,3 & 571 & 4,4 & 7,5 & 4283 \\
\hline Zona 02 & 164,4 & 895 & 5,2 & 13,1 & 11725 \\
\hline
\end{tabular}

Fuente: elaboración propia

Los potenciales de plantíos de árboles frutales en ambos los campos del gradiente social investigado demuestran una posibilidad de refuerzo de la seguridad alimentaria y de conservación de la diversidad biológica, a través de plantíos en los patios, preferencialmente de árboles frutales.

De las especies identificadas en el Jardim Universal y en la Zona 02, nueve están clasificadas como amenazadas de extinción. Dos especies ocurren en los dos barrios analizados: Araucaria angustifolia y Delonix regia. Las especies amenazadas encontradas en los patios poseen bajos índices de valor de preferencia, excepto Euterpe edulis, relativamente diseminada por la Zona 02 (IVP de 9,5\%). Esos resultados demuestran, por lo tanto, la viabilidad de los patios de la región metropolitana de Maringá para prácticas de conservación ex-situ de especies vegetales amenazadas de extinción (cuadros 9 y 10).

Cuadro 9. Especies amenazadas de extinción, Jardim Universal

\begin{tabular}{|l|l|}
\hline Especie & Índice de Valor de Preferencia \\
\hline Araucaria angustifolia & 1,6 \\
\hline Butia eriospatha & 2,4 \\
\hline Delonix regia & 2,9 \\
\hline
\end{tabular}

Fuente: elaboración propia 
Cuadro 10. Especies amenazadas de extinción, Zona 02

\begin{tabular}{|l|l|}
\hline Especie & Índice de Valor de Preferencia \\
\hline Araucaria angustifolia & 1,2 \\
\hline Cupressus macrocarpa & 1,6 \\
\hline Delonix regia & 0,4 \\
\hline Dicksonia sellowana & 2 \\
\hline Euterpe edulis & 9,5 \\
\hline Heliconia angusta & 2 \\
\hline
\end{tabular}

Fuente: elaboración propia

\section{DISCUSIÓN}

Los escores obtenidos en el cálculo del Índice de Diversidad de Shannon en el Jardim Universal y en el barrio Zona 02 son similares a aquellos mensurados en bosques prístinos en sureste de Brasil, que oscilaran entre 3,16 y 4,29 (Cielo Filho y Santin, 2002). Ahora bien, ¿qué significados tienen la riqueza de especies y los demás datos respecto a la flora de patios, que hemos obtenido a través del gradiente social investigado?

Indudablemente, el incremento del número de árboles en los patios debe constituirse en el primer objetivo concreto de planificación, por su escala espacial y temporal de beneficios socio-ambientales. Patios con más árboles están positivamente correlacionados a especies de invertebrados (Loram et al. 2008), muchos de las cuales son especies polinizadoras. Entonces, ¿hay un área mínimo para garantizar la presencia de una mayor densidad arbórea en esos espacios? Esta es una cuestión en abierto respecto la ecología de los patios (Goddard et al. 2010). Mitchell y Hanstad (2004) mensuraron en circa $167 \mathrm{~m}^{2}$ el área crítico para el incremento del número de árboles presentes en patios.

Hemos obtenido correlaciones positivas entre el área medio no encementado de los patios del Jardim Universal $\left(108,3 \mathrm{~m}^{2}\right)$, y asimismo en el barrio Zona 02, donde el área medio no encementado de los patios es de 164,4 $\mathrm{m}^{2}$. La correlación especies-área es aplicable a la escala del patio, y el área usualmente está relacionada a no solo a la riqueza de especies, sino también a la heterogeneidad de coberturas de suelo [número de árboles, polígonos con vegetación ornamental, céspedes, etcétera (Loram et al. 2008; Goddard et al. 2010)]. No obstante, dichas correlaciones no son universales. Angeoletto (2012) en estudio similar realizado en el barrio Conjunto Triangulo (también localizado en la ciudad de Sarandi, región metropolitana de Maringá), verificó no haber correlación entre el área medio de los patios del barrio, de $70 \mathrm{~m}^{2}$, y entre el número de especies presentes, entre el número total de individuos cultivados y entre el número de árboles presentes.

Por lo que recomendamos garantizar, a través de legislación específica, patios con un área mínimo de $110 \mathrm{~m}^{2}$ no pavimentado, disponibles a la flora, en la región metropolitana de Maringá. Patios con áreas reducidos usualmente tienen menos árboles, principalmente individuos con doseles superiores a dos metros, lo que pode significar daños económicos, sociales, estéticos y ecológicos (Loram et al. 2008). Áreas de patios excesivamente reducidos podrían comprometer, por ejemplo, las 
posibilidades de conectividad entre paisajes urbanos y forestales (Díaz et al. 2011), y, por extensión, de un flujo génico entre ellos.

Por el carácter fragmentado de la flora de patios, que está distribuida en teselas de hábitats pequeños y aislados, la correlación positiva estándar entre la riqueza de especies y área, que normalmente ocurre en ambientes prístinos (Odum, 1988) es especialmente importante en sistemas ecológicos urbanos. Teselas de hábitats más grandes soportan poblaciones mayores y más estables de pájaros. Lo mismo pasa con otras taxones que habitan las ciudades, como anfibios, mamíferos y carábidos (Goddard et al. 2010). El número de especies cultivadas, de individuos cultivados e y árboles cultivados se correlacionan positivamente (aunque flacamente) a la edad de las viviendas. Esos datos apuntan un incremento de la diversidad vegetal a lo largo del tiempo. No obstante, recomendamos el desarrollo de proyectos de introducción de especies vegetales de usos diversos, de modo a apresurar una mayor riqueza específica en los patios.

El porcentaje de patios completamente solados en el barrio de baja renta es casi tres veces aquel encontrado en el barrio Zona 02. La práctica de pavimentación de los patios generalmente atrae poca atención por la pequeña escala del cambio, pero las consecuencias pueden alcanzar una magnitud considerable. En la ciudad de Leeds (UK), por ejemplo, entre 1971 y 2004 el aumento de la superficie pavimentada de los patios fue de un $138 \%$, fundamentalmente para la construcción de garajes. Como consecuencia, las inundaciones quedaron más frecuentes, y más intensas (Perry y Nawaz, 2008).

Loram ( et al. 2007), en estudio sobre la vegetación de patios de cinco ciudades del Reino Unido, calcularon que la tendencia de los ciudadanos en solar los jardines frontales de sus viviendas para aparcar coches, podría resultar en la pérdida de hasta $25 \%$ del área disponible para la vegetación, con impactos a la biodiversidad y aumento de las inundaciones en algunas áreas, principalmente aquellas ya vulnerables al fenómeno.

Las decisiones individuales de los vecinos de los barrios investigados, respecto a la impermeabilización de sus patios se constituyen en un ejemplo del fenómeno de la tiranía de las pequeñas decisiones. La expresión inicialmente ha sido acuñada por el economista Alfred E. Kahn, para describir fenómenos económicos en los cuales decisiones, individualmente pequeñas en alcance y perspectiva temporal, cumulativamente resultan en una situación no deseable. Odum (1982) adaptó la premisa a la degradación ambiental.

Afrontar la cuestión de la pavimentación de de los patios es una tarea urgente, y muy compleja. La legislación respecto a es usualmente floja. En la ciudad de Maringá, por ejemplo, hay una ley exigiendo la manutención de por lo menos el 10\% de los áreas de los patios permeable (Angeoletto, 2012). El porcentaje es claramente insuficiente, o bien para la conservación más amplia de la biodiversidad a través de un sistema de áreas verdes en los patios, o bien para absorción de lluvias, en un contexto de incremento 
constante de la infraestructura para la circulación de vehículos privados en las ciudades brasileñas.

La riqueza de especies vegetales en patios urbanos suele ser elevada, aunque corrientemente mal distribuida. Barrios de mayor status socioeconómico normalmente presentan una mayor diversidad vegetal en sus patios, porque tienen más recursos para introducir nuevas especies de acuerdo con sus preferencias personales (Grove et al. 2006). Además estos patios suelen tener más área disponible a la diversificación vegetal (Thompson et al. 2004), como hemos verificado en nuestra comparación de la diversidad vegetal de patios de barrios de diferentes clases sociales. Sin embargo, la riqueza de especies, en nuestros estudios, tanto entre los pobres cuanto entre los vecinos de clase media alta, sigue un estándar: unas pocas especies de alto índice de valor de preferencia (IVP), es decir, comunes en los patios, seguidas por docenas de otras de bajo IVP, un resultado similar al encontrado por diversos autores (Thompson et al. 2004; Smith et al. 2006a; Marco et al. 2008).

De todos modos, aunque la riqueza de especies sea un indicador sencillo de la diversidad vegetal, ella posibilita una pronta comprensión de los parámetros de biodiversidad por especialistas de otras áreas además de la biología, y por gestores públicos (Tzoulas y James, 2010). El establecimiento de un diálogo entre académicos y gestores a partir de datos ecológicos de más fácil compresión es un reto fundamental en el proceso de planificación ambiental urbana, pues, usualmente, gobiernos locales tienen un conocimiento muy limitado de cómo mantener la biodiversidad en ecosistemas urbanos (Sandström et al. 2006).

Patios en algunos casos son puntos de introducción de especies exóticas que, en ambientes prístinos, se tornan invasoras. En el territorio francés, 21 especies vegetales invasoras han sido introducidas a partir de cultivos en patios, y el $90 \%$ de las plantas invasoras de la región mediterránea son especies ornamentales que lograron escapar de los límites de los jardines, y colonizar nuevos hábitats (Marco et al. 2008). Con todo, como la vegetación de los patios se distribuye típicamente como un pool de especies con unas pocas muy abundantes, y muchas con bajas poblaciones (es decir, muchas especies con bajo IVP), es probable que las oportunidades para la mayoría de las especies en colonizar hábitats externos a los jardines sean bastante escasas (Smith et al. 2006a).

El bajo IVP de la mayoría de las especies identificadas en la Zona 02 y en el Jardim Universal podría ser un factor de restricción a la alimentación de herbívoros más selectivos, como algunas especies de insectos. Pero sin embargo, una proporción elevada de herbívoros - incluso insectos - están adaptados a alimentarse de plantas en niveles taxonómicos más elevados, como el género, o mismo la familia (Smith, et al. 2006a).

A su vez, el bajo índice de similitud de especies encontrado, hecho que indica alta diversidad beta a lo largo del gradiente social estudiado, debe de ser mantenido a través del incentivo al plantío de especies distintas a las encontradas en los barrios, preferencialmente especies sin ocurrencia en los patios estudiados. Además de 
conservarse la diversidad beta, la introducción de nuevas especies aumentaría el índice de diversidad de Shannon-Wiener, y se evitaría que una o unas pocas especies se tornaran dominantes, disminuyéndose así la posibilidad de invasión biológica por parte de especies vegetales oriundas de los patios urbanos.

Hay una tendencia bien definida de predominio de cultivos de especies utilitarias entre los pobres, y de especies ornamentales entre vecinos de mayor status socioeconómico (Peyre et al. 2006; Lubbe et al. 2010), y la hemos detectado también en nuestra investigación. En consonancia con su estilo de vida, los vecinos pobres del barrio Jardim Universal disponen de menos recursos (energéticos, materiales, monetarios y técnicos), y menos área en la gestión de la vegetación de sus patios. No hay entre ellos una ecología del prestigio, materializada en una abundancia de plantas ornamentales ordenadas a través de inputs de arquitectura paisajística, como es común en barrios de clase media alta (Grove et al. 2006), sino suelo disponible para la posibilidad de expansión de sus viviendas. Hope (et al. 2003) denominan de efecto lujuria al fenómeno - los más ricos y escolarizados cercándose de vegetación - y creando paisajes en sus hogares con la flora.

Como demuestran nuestros datos, la desigualdad al acceso a la vegetación configura con claridad una situación de injusticia ambiental entre las distintas clases sociales, fenómeno que implica en la distribución no ecuánime de la vegetación a través de los barrios, lo que se refleja en peores condiciones de vida (Pedlowski et al. 2003; Perkins et al. 2004). Se trata de un fenómeno común en las urbes brasileñas. En la ciudad de São Paulo por ejemplo, Lombardo (1985), al estudiar la geografía de las islas de calor, observó que el acceso a la vegetación es menor cuanto más pobre sea el barrio. La misma situación se puede observar en otras ciudades, como Ribeirão Preto (Guzzo, 1999). También en otros países acentuadamente desiguales, como África del Sur, iniquidades en el acceso a la vegetación caracterizan las ciudades (Lubbe et al. 2010).

Efectivamente, la desigualdad en el acceso a la flora y sus beneficios es incluso más grave en el Jardim Universal, una vez que, diferentemente del barrio Zona 02, donde hay dos parques próximos, no existe allí ninguna área verde, hecho que aumenta sustancialmente la importancia del aumento de la cobertura vegetal, principalmente aquella de carácter arbóreo y arbustivo. Efectivamente, patios son estratégicos al incremento de áreas verdes en barrios donde hay escasez de vegetación (Rudd et al. 2002). No se trata meramente de una cuestión estética: varios estudios correlacionan barrios abundantemente vegetados a una menor incidencia de diversos tipos de enfermedades, como las respiratorias (Takano et al. 2002; Tzoulas et al. 2007).

Se puede dirimir la injusticia ambiental entre barrios de la región metropolitana de Maringá a través de proyectos de plantíos de especies vegetales arbóreos. Por cierto, esos programas de introducción de especies deben de ser precedidos por investigaciones de carácter sociológico, con el objetivo de identificarse las maneras de cómo la vegetación nativa - en general, menos conocida que especies exóticas - puede ser aceptada e incorporada a los patios (Kendal et al. 2010). El éxito de programas de plantíos de árboles depende crucialmente de la aceptación pública, e involucrar la 
sociedad civil en acciones de plantíos es una tarea compleja, $\mathrm{y}$, usualmente, lenta (Ames, 1980; Angeoletto, 2008; Angeoletto,

2012).

En Brasil, la seguridad alimentaria es una cuestión trans-social. Según el Instituto Brasileiro de Geografia e Estatística (IBGE, 2011) menos de 10\% de los brasileños con 10 años o más consumen diariamente los 400 gramos de frutas y hortalizas recomendados pela Organización Mundial de la Salud. Estudios epidemiológicos indican que el consumo abundante de frutas y verduras está asociado a bajas incidencias de varios tipos de cáncer, enfermedades cardiovasculares, enfermedades degenerativas relacionadas a vejez, y enfermedades oculares, como la catarata (Padovani, 2003).

En este sentido, una importante función de los patios, que enlaza la biología de la conservación y la seguridad alimentaria, es la manutención de variedades de árboles frutales y otras especies de carácter alimenticio que no son cultivadas por la agricultura industrial. Esas especies y variedades frecuentemente poseen alelos raros (formas alternativas de un mismo gene) (Negri, 2003), hecho que justifica aun más la conservación de la agrobiodiversidad in vivo en los patios. Efectivamente, patios urbanos son importantes sitios de agrobiodiversidad (Siviero et al. 2011), y su manutención es importantes para la obtención de nuevas variedades de cultivos más adaptables a los cambios ambientales globales (Galluzzi et al. 2010).

\section{CONSIDERACIONES FINALES}

En Brasil los patios suponen centenares de hectáreas en las ciudades, espacios disponibles para recibir la vegetación que contribuya a una mayor seguridad alimentaria y calidad de vida de los ciudadanos. Los patios pueden además apoyar la conservación ex-situ, albergando especies en peligro de extinción como es el caso de Araucaria angustifolia, especie críticamente amenazada de extinción y cuyas semillas son muy apreciadas en la cultura alimentaria del sur de Brasil. A pesar de su potencial, los patios son invisibles a las autoridades municipales. No existe en las ciudades de la región metropolitana de Maringá legislación específica, ni datos sistematizados que permitan la planificación y gestión para el incremento de la vegetación en esos espacios - nuestro estudio ha sido pionero en el objetivo de dibujarse un cuadro de la diversidad vegetal en patios de diferentes clases sociales, en la RMM.

Hay espacio para un continuum de patios vegetados a lo largo del gradiente social investigado. Hecho inusual en las periferias pobres de las ciudades brasileñas, caracterizadas por una considerable escasez de suelo (Angeoletto, 2008; Angeoletto, 2012), los patios del barrio Jardim Universal son peculiares por presentaren áreas medias no pavimentadas que ultrapasan los $100 \mathrm{~m}^{2}$. Para la mayor parte de las familias urbanas pobres del planeta, la escasez de suelo es el principal obstáculo para el establecimiento de patios abundantemente vegetados (Mitchell y Hanstad, 2004), y plantíos de árboles en sus patios podrían disminuir el estado de injusticia ambiental en que se encuentran los vecinos del Jardim Universal. Esos plantíos podrían también representar un refuerzo a la seguridad alimentaria, o bien a los vecinos más pobres, o bien a los de clase media alta. Como hemos observado, la inseguridad alimentaria en Brasil es una cuestión trans-social. 
Los patios del Jardim Universal son la única posibilidad de introducción de áreas verdes, a través de plantíos de árboles. Como hemos demostrado, su potencial de plantíos es elevado - se podrían plantar en los patios del barrio aproximadamente 4.300 árboles. En el barrio Zona 02 el potencial de plantíos es aun más elevado - se podrían introducir en sus patios circa 12.000 árboles. Plantíos de árboles son la mejor manera de aumentarse la abundancia de una amplia gama de taxones de invertebrados y vertebrados en patios urbanos (Smith et al. 2006a; Loram et al. 2008). Además, en los árboles, la fauna silvestre está más protegida contra la depredación por animales domésticos, como Felis catus (Baker et al. 2005).

En este sentido, abogamos la necesidad de una planificación que contemple el eventual incremento del área construida de las viviendas, en paralelo al mantenimiento del área disponible para plantíos, principalmente de especies arbóreas. Esto se podría alcanzar, por ejemplo, a través de la verticalización de las viviendas aliada a la introducción de árboles en los patios, en proyectos conducidos por los ingenieros, arquitectos y biólogos de las municipalidades.

La expansión vertical puede incluso abaratar los costes de construcción. Hay técnicas de construcción de pisos que no utilizan ladrillos, sino alambres de acero tranzados, más ligeros y más baratos. Tecnologías semejantes podrían ser usadas en la verticalización de las viviendas. Sin embargo, no sólo el área libre de los terrenos puede ser usado para el incremento de la diversidad vegetal, sino también los tejados y muros. Los tejados verdes consisten básicamente de una camada de vegetación, una de substrato, y una camada de drenaje del exceso de agua de las lluvias. Además de aumentaren la diversidad vegetal (se podría cultivar incluso alimentos), la capacidad de los tejados verdes en dirimir posibles inundaciones en áreas muy impermeabilizadas es un hecho (Mentens et al. 2006).

A su vez, los muros que dividen las viviendas representan centenares de metros cuadrados disponibles al plantío de especies de lianas como las ornamentales Antigonon leptopus, Parthenocissus tricuspidata, Allamanda cathartica y otras, inclusive especies alimentarias, como Sechium edule y Passiflora spp. Un terreno de 40 metros de extensión, con dos muros de tres metros de altura separándolo de sus vecinos, añade, en una propiedad, $240 \mathrm{~m}^{2}$ de área disponible para plantíos. En tiempos en que se aboga la disminución del área urbanizable, y la densificación poblacional, a esas extensiones laterales no se pueden encararlas como meros límites. Además del efecto paisajístico, y de la atenuación de las islas de calor, esas superficies pueden ser proyectadas para la nidificación de especies de pequeños pájaros silvestres que ocurren en sistemas ecológicos urbanos, como Troglodytes musculus, a través de cavidades en los muros.

Con todo, una planificación más exitosa de los patios urbanos sólo será posible con estudios previos que logren comprender factores ambientales, culturales y socioeconómicos que influyen en su configuración. En ese contexto, la metodología que hemos empleado para comparar los barrios podría ser utilizada para evaluarse las condiciones bioestructurales de esos hábitats, en otros estudios de caso. 
Como cantó el bardo Bob Dylan, y le pedimos permiso para una paráfrasis, we've stepped in the middle of seven sad forests. We've been out in front of a dozen dead oceans. We've been ten thousand miles in the mouth of a graveyard. Tras millares de años e incontables aventuras y sacrificios, hemos llegado a las ciudades, nuestro definitivo hogar. Nosotros deseamos, luego, existimos. Con la fuerza del deseo de muchos podremos cambiar algunas de las grises realidades de hoy, y hacer de las ciudades brasileñas puntos calientes de diversidad biológica, y plenas de justicia ambiental. Lujurias verdes, mimetizadas en entornos igualmente verdes. No las tristes megalópolis presentadas en obras de ciencia ficción como la película Blade Runner, sino ciudades menores. Se tuviéremos éxito en llegar a ese punto de sofisticación biológica, entonces habremos comprendido que lo importante es que perdamos importancia. Y que perder importancia significa apagar las huellas de las ciudades sobre la biosfera, e dejar que en el inmenso palco escénico que es la Naturaleza, se desarrolle la gran obra teatral de la evolución de la vida.

\section{AGRADECIMIENTOS}

Agradecemos a CAPES y al Ministerio de Ciencia y Tecnología del gobierno español, cuya financiación posibilitó la realización de la investigación descripta en este artículo [Proyecto Ecología Urbana en Regiones Metropolitanas de Brasil: Paisaje, Calidad de Vida y Desarrollo Humano (ECOURBE), del Plan Nacional de I+D+i (CSO2009-12689)]. Y a los agricultores urbanos Helena Stern y Lajar Muzuris, por su fundamental apoyo a nuestros estudios sobre la ecología de las ciudades.

\section{REFERENCIAS}

AMES, R.G. The Sociology of Urban Tree Planting. Journal of Arboriculture 6(5):120-123. 1980.

ANGeOletTO, F. Pelos Quintais de Sarandi: Ecologia Urbana e Planejamento Ambiental. Editora da Universidade Estadual de Maringá. Maringá, Brasil, 2008.

ANGEOLETTO, F. Planeta Ciudad: Ecología Urbana y Planificación de Ciudades Medias de Brasil. Tesis doctoral. Doctorado en Ecología y Medio Ambiente de la Universidad Autónoma de Madrid. Madrid, 2012.

BAKER, P.J.; BENTLEY, A.J.; ANSELL, R.J. (et al.). Impact of predation by domestic cats Felis catus in an urban area. Mammal Review 35: 302-312. 2005.

BOTKIN, D.B.; BEVERIDGE, C.E. Cities as environments. Urban Ecosystems 1: 3-19. 1997.

CARVALHO, E. Exclusão social e crescimento das cidades médias brasileiras. Scripta Nova Revista Electrônica de Geografía y Ciencias Sociales 6(146): 234-245. 2003.

CHAPIN III, F.S.; ZAVALETA, E.S.; EVINER, V.T., (et al.). Consequences of changing biodiversity. Nature 405: 234-242, 2000.

CIELO FILHO, R.; SANTIN, D.A. Estudo florístico e fitossociológico de um fragmento florestal urbano - Bosque dos Alemães, Campinas, SP. Revista Brasileira de Botânica 25(3): 291-301. 2002.

COHEN, B. Urbanization in Developing Countries: Current Trends, Future Projections, and Key Challenges for Sustainability. Technology in Society 28(1-2): 63-80. 2006. 
DANIELS G.D.; KIRKPATRICK, J.B. Comparing the characteristics of front and back domestic gardens in Hobart, Tasmania, Austrália. Landscape and Urban Planning 78: 344-352, 2006.

DAVIES, Z.G.; FULLER, R.A.; LORAM, A. A national scale inventory of resource provision for biodiversity within domestic gardens. Biological Conservation 142: 761-771. 2009.

DÍAZ, F.; QUÉTIER, F.; CÁCERES, D.M. (et al.). Linking Functional Diversity and Social Strategies in a Framework for Interdisciplinary Analysis of Nature's Benefits to Society. Proceedings of the National Academy of Sciences 108(3) 895-902. 2011.

DOW, K. Social dimensions of gradients in urban ecosystems. Urban Ecosystems 4: 255-275. 2000.

FLORENTINO, A.T.N.; ARAÚJO, E.L.; ALBUQUERQUE, U.P. Contribuição de pátios agroflorestais na conservação de plantas da caatinga, município de Caruaru, PE, Brasil. Acta Botanica Brasilica 21(1): 37-47, 2007.

GALLUZZI, G.; EYZAGUIRRE, P.; NEGRI, V. Home gardens: neglected hotspots of agrobiodiversity and cultural diversity. Biodiversity \& Conservation 19(13): 3635-3654, 2010.

GASTON, K.J.; WARREN, P.H.; THOMPSON, K. (et al.). Urban domestic gardens (IV): the extent of the resource and its associated features. Biodiversity and Conservation 14: 3327-3349, 2005.

GODDARD, M.A.; DOUGILL, A.J.; BENTON, T.G. Scaling up from gardens: biodiversity conservation in urban environments. Trends in Ecology and Evolution 25: 90-98, 2010.

GONZÁLEZ-GARCÍA, A.; GÓMEZ-SAL, A. Private Urban Greenspaces or "Patios" as a Key Element in the Urban Ecology of Tropical Central America. Human Ecology 36: 291-300, 2008.

GRIMM, N.B.; GROVE, J.M.; PICKETT S.T.A. (et al.). Integrated approaches to long-term studies of urban ecological systems. BioScience 50: 571-584. 2000.

GRIMM, N.B.; REDMAN, C.L. Approaches to the study of urban ecosystems: The case of Central Arizona-Phoenix. Urban Ecosystems 7: 199-213. 2004.

GRIMM, N.B.; FAETH, S.H.; GOLUBIEWSKI, N.E. (et al.). Global change and the ecology of cities. Science 319(5864): 756-760. 2008.

GROVE, J.M.; TROY, A.R.; O'NEIL-DUNNE, J.P.M. (et al.). 2006. Characterization of households and its implications for the vegetation of urban ecosystems. Ecosystems 9: 578-597. 2006.

GUZZO, P. Estudo dos espaços livres de uso público da cidade de Ribeirão Preto/SP, com detalhamento da cobertura vegetal e áreas verdes de dois setores urbanos. Dissertação de Mestrado. Mestrado em Geociências e Meio Ambiente. Universidade Estadual Paulista. Rio Claro, Brasil, 1999.

HAEUBER, R.; RINGOLD, P. Ecology, the social sciences, and environmental policy. Ecological Applications 8: 330-331. 1998.

HOPE, D.; C. GRIES, C.; ZHU, W. (et a.l). Socioeconomics drive urban plant diversity. Proceedings of National Academy of Sciences 100(15): 8788-3792. 2003.

IBGE. Pesquisa de Orçamentos Familiares 2008-2009 - Análise do Consumo Alimentar Pessoal no Brasil. IBGE. Rio de Janeiro, Brasil. 2011. 
KENDAL, D.; NICHOLAS, S.G.; WILLIAMS, J.H. (et al.). Harnessing diversity in gardens through individual decision makers. Trends in Ecology and Evolution 25(4): 201-202. 2010.

LOMBARDO, M. A. Ilha de Calor nas Metrópoles: o exemplo de São Paulo. Editora. Hucitec. São Paulo, Brasil, 1985.

LORAM, A.; TRATALOS, J.; WARREN, P.H. (et al.). Urban domestic gardens (X): the extent \& structure of the resource in five major cities. Landscape Ecology 22: 601-615, 2007.

LORAM, A.; WARREN, P.H.; GASTON, K.J. Urban Domestic Gardens (XIV): The Characteristics of Gardens in Five Cities. Environmental Management 42: 361-376, 2008.

LUBBE, C.S.; SIEBERT, S.J.; CILLIERS, S.S. Political legacy of South Africa affects the plant diversity patterns of urban domestic gardens along a socio-economic gradient. Scientific Research and Essays 5(19): 2900-2910, 2010.

MARCO A.; DUTOIT, T.; DESCHAMPS-COTIM, M. (et al.). Gardens in urbanizing rural areas reveal an unexpected floral diversity related to housing density. Comptes Rendus Biologies 331(6): 452-465, 2008.

MCDONNELL; M.J.; HAHS, A.K. The use of gradient analysis studies in advancing our understanding of the ecology of urbanized landscapes: current status and future directions. Landscape Ecology 23: 1143-1155. 2008.

MENTENS, J.; RAES, D.; HERMY, M. Green roofs as a tool for solving the rainwater runoff problem in the urbanized 21st century? Landscape and Urban Planning 77: 217-226. 2006

MITCHELL, R.; HANSTAD, T. Small Homegardens Plots and Sustainable Livelihoods for the Poor. FAO LSP WP 11, Access to Natural Resources Sub-Programme, 2004.

MONTGOMERY, M.R. The Urban Transformation of the Developing World. Science 319(8): 761-764. 2008.

NEGRI, V. Landraces in central Italy: where and why they are conserved and perspectives for their on-farm conservation. Genetic Resources and Crop Evolution 50: 871-885. 2003.

ODUM, E.P. Ecologia. Editora Guanabara Koogan. Rio de Janeiro, Brasil. 1988.

ODUM, W.E. Environmental Degradation and the tyranny of small decisions. Bioscience 32(9): 728-729. 1982.

PADOVANI, R.M. Disponibilidade de carotenóides em relação à energia e proteínas nos domicílios de famílias das regiões metropolitanas brasileiras. Dissertação de Mestrado. Mestrado em Alimentos e Nutrição. Unicamp. Campinas, Brasil. 2003.

PEDLOWSKI, M.A.; SILVA, V.A.; ADELL J.J. (et al.). Urban forest and environmental inequality in Campos dos Goytacazes, Rio de Janeiro, Brazil. Urban Ecosystems 6: 9-20, 2003.

PERKINS, H.A.; HEYNEN, N.; WILSON, J. Inequitable access to urban reforestation: the impact of urban political economy on housing tenure and urban forests. Cities 21(4): 291-299, 2004.

PERRY, T.; NAWAZ, R. An investigation into the extent and impacts of hard surfacing of domestic gardens in an area of Leeds, United Kingdom. Landscape and Urban Planning 86: 113. 2008. 
PEYRE A.; GUIDAL, A.; WIERSUM K.F. (et al.). Dynamics of homegarden structure and function in Kerala, India. Agroforestry Systems 66: 101-115. 2006.

PICKETT, S.T.A.; BURCH, W.R.; DALTON, S.E. A conceptual framework for the study of human ecosystems in urban areas. Urban Ecosystems 1(4): 185-199. 1997.

PICKETT S.T.A.; GROVE, M. Urban ecosystems: What would Tansley do? Urban Ecosystems 12(1): 1-8. 2009.

PNUMA (Programa de las Naciones Unidas para el Medio Ambiente). Anuario PNUMA Temas Emergentes en Nuestro Medio Ambiente Global. PNUMA. Nairobi, Kenya. 2011.

ROBBINS, P.; POLDERMAN, A.; BIRKENHOLTZ, T. Lawns and Toxins: An Ecology of the City. Cities 18(6): 369-380. 2001.

ROSENZWEIG, M.L. Loss of speciation rate will impoverish future diversity. Proceedings of the National Academy of Sciences 98(10): 5404-5410. 2001.

RUDD, H.; VALA, J.; SCHAEFER, V. Importance of backyard habitat in a comprehensive biodiversity conservation strategy: a connectivity analysis of urban green spaces. Restoration Ecology 10: 368-375. 2002.

SANDSTRÖM, U.G.; ANGELSTAM, P.; KHAKEE, A. Urban comprehensive planning identifying barriers for the maintenance of functional habitat networks. Landscape and Urban Planning 75: 43-57. 2006.

SANTOS, C. Estatística Descritiva - Manual de Auto-aprendizagem. Edições Sílabo. Lisboa, Portugal, 2007.

SCHAEDEK, U.; STRAUSS, B.; BIERDERMANN, R. (et al.). Plant species richness, vegetation structure and soil resources of urban brownfield sites linked to successional age. Urban Ecosystems 12: 115-126. 2009.

SIVIERO, A.; DELUNARDO, T.A.; HAVERROTH, M. (et al.). Cultivo de espécies alimentares de Rio Branco, Acre, Brasil. Acta Botanica Brasilica 25(3): 549-556. 2011.

SMITH, R.M.; THOMPSON, K.; HODGSON, J.G. (et al.). Urban domestic gardens (IX): Composition and richness of the vascular plant flora, and implications for native biodiversity. Biological Conservation 129: 321-322, 2006 .

SMITH, R.M.; WARREN, P.H.; THOMPSON, K. (et al.). Urban domestic gardens (VI): environmental correlates of invertebrate species richness. Biodiversity and Conservation 15: 2415-2438, 2006b.

TERRADAS, J. Ecología Urbana. Editorial Rubes. Barcelona, España. 2001.

TAKANO, T.; NAKAMURA, K.; WATANABE, M. Urban residential environments and senior citizens' longevity in mega-city areas: the importance of walkable green space. Jounal of Epidemiology \&. Community Health 56(12): 913-916, 2002.

THE ANGIOSPERM PHYLOGENY GROUP. An update of the Angiosperm Phylogeny Group classification for the orders and families of lowering plants: APG III. Botanical Journal of the Linnean Society 161: 105-121. 2009.

THOMPSON, K.; HODGSON, J.G.; SMITH, R.M. (et al.). Urban domestic gardens (III): Composition and diversity of lawn floras. Journal of Vegetation Science 15: 373-378, 2004. 
THOMPSON, K.; COLSELL, S.; CARPENTER, J. (et al.). Urban domestic gardens (VII): a preliminary survey of soil seed banks. Seed Science Research 15: 133-141, 2005.

TZOULAS, K.; KORPELA, K.; VENN, S. (et al.). Promoting ecosystem and human health in urban areas using Green Infrastructure: A literature review. Landscape and Urban Planning 81: 167-178, 2007.

TZOULAS, K.; JAMES, P. Making biodiversity measures accessible to non-specialists: an innovative method for rapid assessment of urban biodiversity. Urban Ecosystems 13: 113-127. 2010.

UNFPA. Estado de la Población Mundial 2007. United Nations Population Fund. New York, USA, 2007.

UNITED NATIONS. (Department of Economic and Social Affairs/Population Division) World Urbanization Prospects: The 2007 Revision. New York, USA. 2008.

VITOUSEK, P.M. Beyond global warming: ecology and global change. Ecology 75: 1861-1876, 1994. 